\title{
Bioprosthetic valve replacement in idiopathic hypereosinophilic syndrome with mitral valve involvement
}

\section{Qin Fang, MD, Tian-Xiang Gu, MD, Zong-Yi Xiu, MD, and Zhi-Wei Zhang, MD, Shenyang City, China}

Idiopathic hypereosinophilic syndrome (IHES) is a leukoproliferative disorder characterized by cytokine-induced overproduction of eosinophils, with resultant multiorgan infiltration and damage. The diagnostic criteria include evidence of end organ damage, exclusion of all other causes of eosinophilia, and sustained absolute eosinophil count greater than $1.5 \times 10^{9}$ cells/L for at least 6 months. ${ }^{1}$

From the Department of Cardiac Surgery, First Hospital of China Medical University, Shenyang City, China.

Disclosure: Authors have nothing to disclose with regard to commercial support. Received for publication June 11, 2013; revisions received July 7, 2013; accepted for publication July 12, 2013; available ahead of print Sept 3, 2013.

Address for reprints: Tian-Xiang Gu, MD, No. 155, Nanjing N St, Heping District,

Shenyang City, Liaoning Province, China (E-mail: fangqinok@163.com).

J Thorac Cardiovasc Surg 2013;146:e45-7

$0022-5223 / \$ 36.00$

Copyright $(\underset{)}{ } 2013$ by The American Association for Thoracic Surgery

http://dx.doi.org/10.1016/j.jtcvs.2013.07.033
IHES with cardiac involvement often causes morbidity and mortality. It is characterized by endocardial fibrosis and overlying thrombus, leading to restrictive cardiomyopathy and valvular dysfunction. The atrioventricular valves are frequently involved, causing valvular regurgitation.

\section{CLINICAL SUMMARY}

A 36-year-old man with a diagnosis of IHES reported weakness, fever, palpitations, and dyspnea. In the months before his final admission, the patient was admitted to the hospital 5 times, with similar symptoms.

In a first admission, his peripheral blood smears showed a markedly elevated eosinophil count $\left(>8.50 \times 10^{9}\right.$ cells $/ \mathrm{L}$, $60.6 \%$ in the differential leukocyte count). Transthoracic echocardiography (TTE) showed posterior leaflet mitral valve heteroplasia and moderate mitral regurgitation. In a second
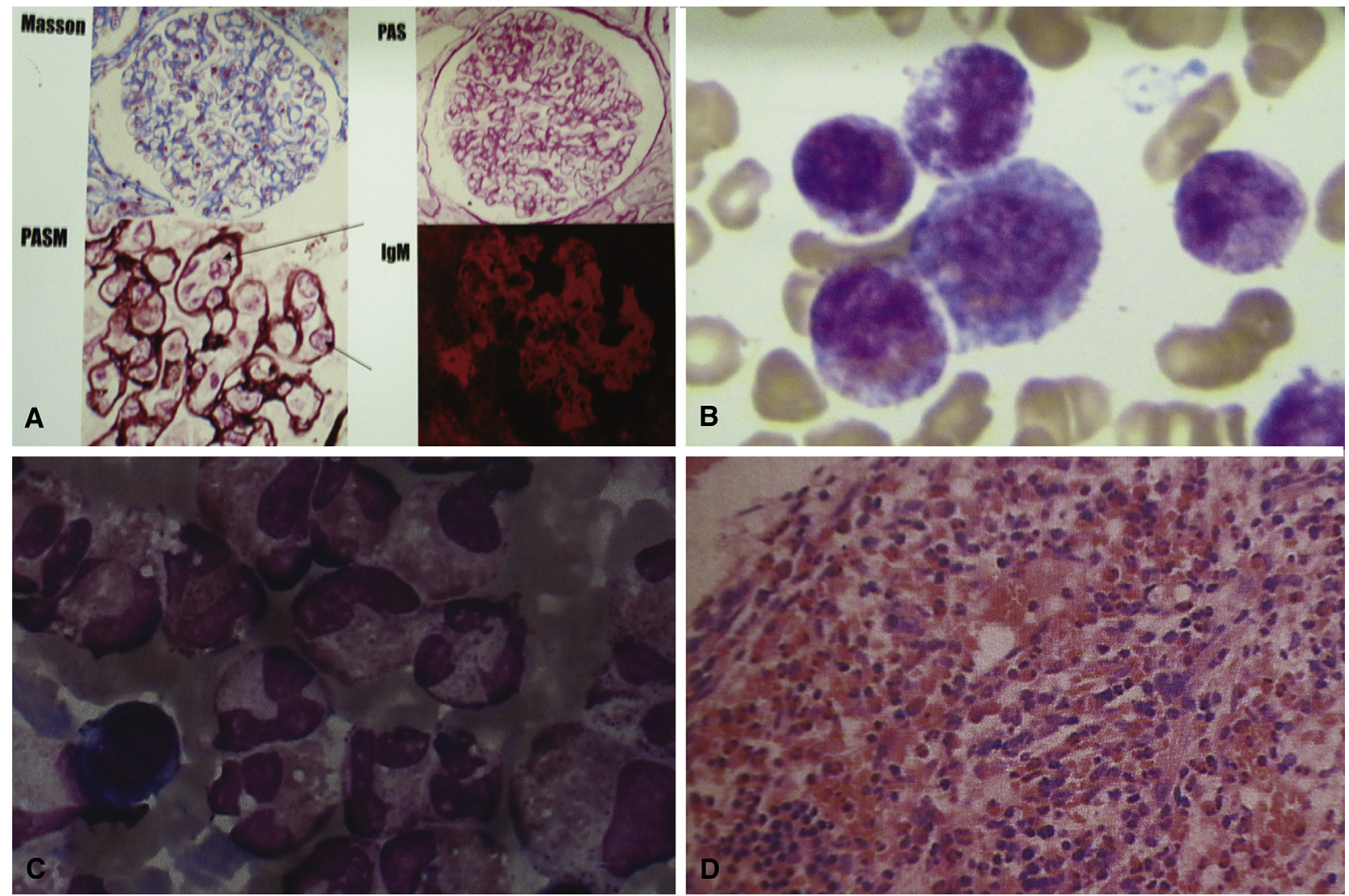

FIGURE 1. A and B, Second admission. A, Renal biopsy sample shows convalescence of endocapillary proliferative glomerulonephritis, but no eosinophilic infiltration. Masson, Masson trichrome stain; PAS, periodic acid-Schiff stain; PASM, periodic Schiff-methenamine stain; IgM, immunoglobulin M. B, Bone marrow aspiration shows granulocyte series active proliferation with an elevated eosinophil ratio. C and D, Third admission. C, Bone marrow aspiration biopsy sample shows granulocyte series active proliferation. D, Bone marrow aspiration biopsy reveals the percentage of eosinophils to be greater than $17 \%$ among other cells seen. 

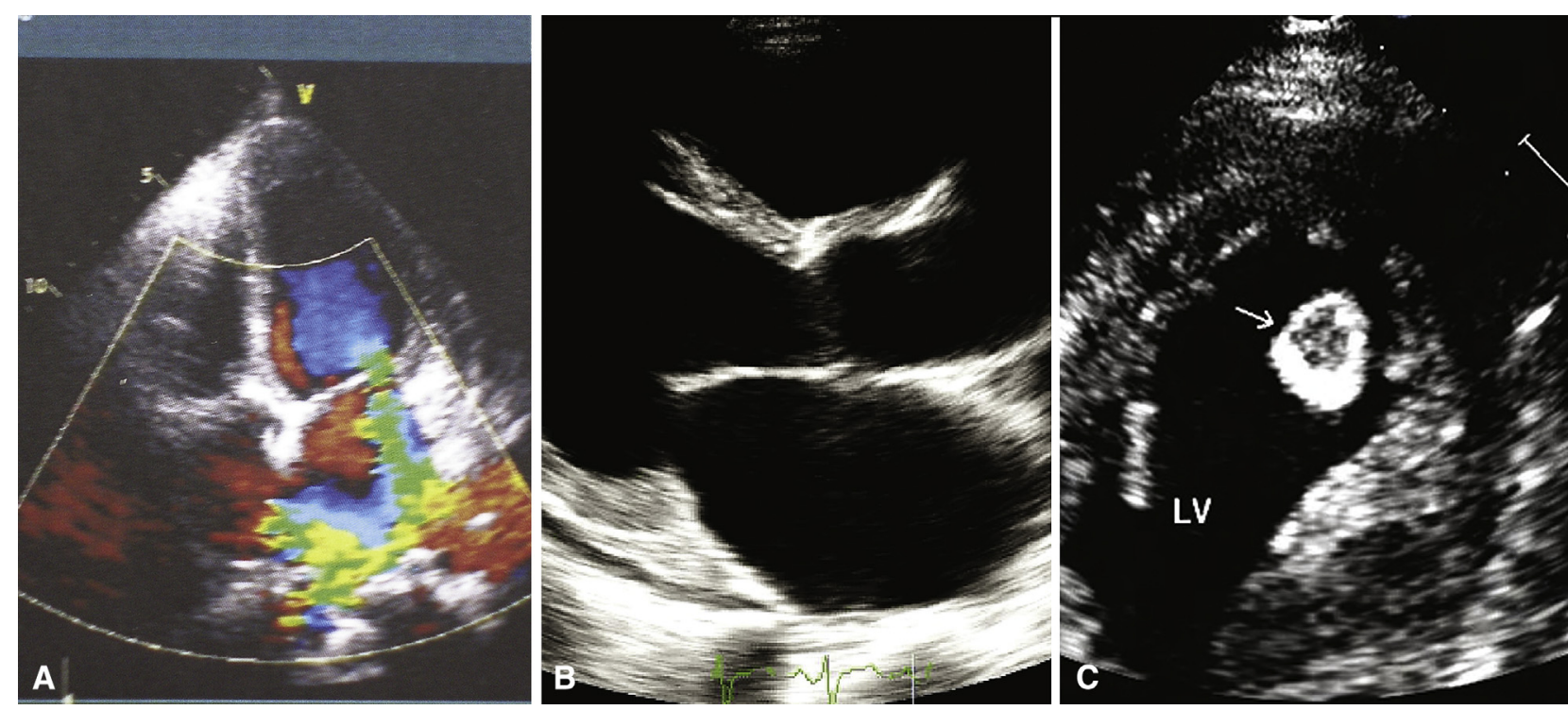

FIGURE 2. A, Transthoracic echocardiography from the third admission shows posterior leaflet of mitral valve heteroplasia and severe mitral regurgitation. B, Transthoracic echocardiography from the fifth admission shows posterior leaflet mitral valve heteroplasia, severe mitral regurgitation, and increased echodensity of the left ventricle. C, Transthoracic echocardiography from the fifth admission shows the bioprosthetic valve to be in good condition; however, the left ventricular $(L V)$ apex is attached with 1 hyperechoic group about $1 \mathrm{~cm}$ in diameter (arrow). There is significant activity.

admission, about 1 month after the first, renal biopsy showed convalescence of endocapillary proliferative glomerulonephritis, but no eosinophilic infiltration was found (Figure 1, A). Bone marrow aspiration showed granulocyte series active proliferation with an elevated eosinophil ratio (Figure 1,B). At a third admission 2 months after the second discharge, bone marrow aspiration biopsy showed granulocyte series active proliferation and revealed the percentage of eosinophils to be greater than $17 \%$ among other cells seen (Figure 1, C and $D$ ). TTE showed posterior leaflet of mitral valve heteroplasia and severe mitral regurgitation (Figure 2, $A$ ). Oral therapy with prednisolone $(60 \mathrm{mg} / \mathrm{d}$ for 1 week, gradually decremented in 2 months) was begun. Cyclosporine (INN ciclosporin, $150 \mathrm{mg} / \mathrm{d}$ ) was used in the next 3 months and then changed back to prednisolone ( $20 \mathrm{mg} / \mathrm{d}$ for 2 months). In the fourth hospitalization, chest computed tomography showed the left chest cavity to be full of pleural effusion.

Before the fifth admission, the patient had stopped taking oral prednisolone for almost 2 months. Blood eosinophilic count also exceeded normal value (percentage of eosinophils to be greater than $12.6 \%$ among white blood cells). Cardiac function was scored as New York Heart Association functional class III to IV. TTE showed posterior leaflet mitral valve heteroplasia, severe mitral regurgitation, and increased echodensity of the left ventricle (Figure 2, B). The patient underwent mitral valve replacement (MVR) with a bioprosthesis (Hancock II; Medtronic, Inc, Minneapolis, Minn). During the operation, we found anterior leaflet prolapse of the mitral valve (Figure 3,A), with posterior leaflet and incapable dissociated chordae tendineae clinging to the posterior wall of the left ventricle (Figure 3, B). In light of the possibilities of infection and influence on wound healing, the patient was not given steroids or chemotherapeutic agents to normalize the peripheral eosinophil count before leaving hospital. His cardiac function returned to New York Heart Association functional class I to II, and had a normal lifestyle after discharge. Mitral valve pathology showed proliferation of fibrous tissue.
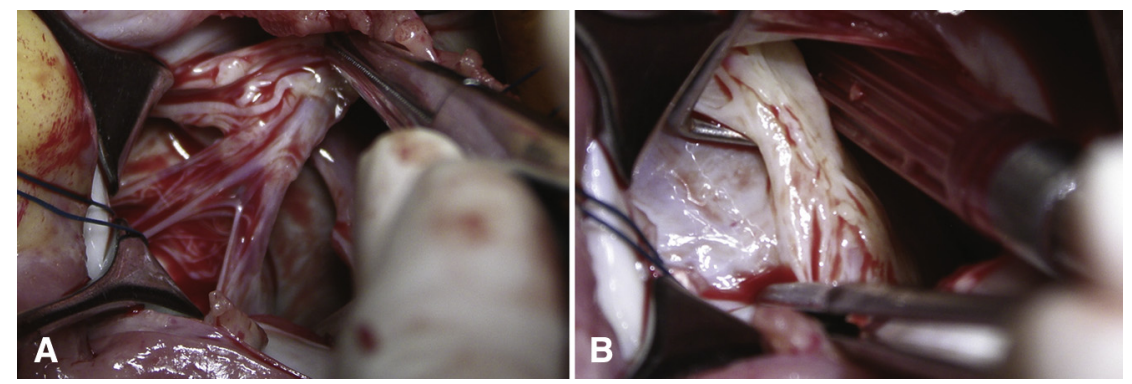

FIGURE 3. Intraoperative photographs show anterior leaflet prolapse of the mitral valve (A) and posterior leaflet and incapable dissociated chordae tendineae clinging to the posterior wall of the left ventricle (B). 
At his final admission, 2 years after MVR, he returned for fever and oliguria of nearly 2 weeks' standing. Routine blood tests showed the total number of leukocytes to be $2.3 \times 10^{9}$ cells/L. Neutrophil ratio was $78.3 \%$, and blood eosinophilic count was normal. The serum creatinine value was $142 \mu \mathrm{mol} / \mathrm{L}$. TTE showed the bioprosthetic valve to be in good condition; however, the left ventricular apex was attached with a single hyperechoic group about $1 \mathrm{~cm}$ in diameter. It had significant activity (Figure 2,C). This time, infective endocarditis was diagnosed. Although blood bacterial culture results were negative, we used cephalosporins and vancomycin to treat empirically. One week after admission, the patient's state was getting worse. Serum creatinine value reached $417 \mu \mathrm{mol} / \mathrm{L}$. Worse, he suddenly became comatose. Brain computed tomographic scan showed cerebral infarction next to the posterior horn of the left frontal lateral ventricle. The patient appeared to be in respiratory failure, and we had to use ventilator therapy. Unfortunately, he died of multiple organ failure 31 hours later.

\section{DISCUSSION}

Cardiac involvement in IHES is characterized by necrosis from infiltration of eosinophils and thrombus formation and, in the late stage, by fibrosis and chronic valvular regurgitation. ${ }^{3}$ Surgical intervention for progressive valvular dysfunction may become necessary. The experience with valve replacement in patients with IHES is limited by the rarity of the disease. Damaged valves can be replaced with mechanical or bioprosthetic valves. Despite anticoagulation, however, mechanical valve replacement may carry a high incidence of thrombosis, whereas bioprosthetic valves may require more frequent replacement for deterioration. In the limited number of patients reported with mechanical valve obstructive thrombosis, reoperation was associated with high mortality. Obstruction of mechanical valves may occur rapidly and in some cases has been reported to develop within days to months of surgery. ${ }^{4}$ Because of these concerns, the use of bioprosthetic valves has been advocated. ${ }^{5}$

This case is significant because it fulfills all the main criteria of IHES. In our case, we prescribed therapy with prednisolone and cyclosporine before surgical intervention. Prednisolone and cyclosporine did not appear to resolve the cardiac valve involvement, the eosinophil counts in peripheral blood and bone marrow, or the symptoms. After MVR with a bioprosthesis, the patient had a normal lifestyle. What is interesting is not only the rarity of the case but the rewarding response in relief of symptoms and a possible cure. Unfortunately, the patient died of infective endocarditis-associated complications 2 years after MVR. This may have been associated with long-term steroid or chemotherapeutic agent use, causing the patient's immune function disorders.

\section{References}

1. Osowo A, Fetten J, Navaneethan S. Idiopathic hypereosinophilic syndrome: a rare but fatal condition presenting with common symptoms. South Med J. 2006;99: $188-9$.

2. Harley JB, McIntosh CL, Kirklin JJ, Maron BJ, Gottdiener J, Roberts WC, et al Atrioventricular valve replacement in the idiopathic hypereosinophilic syndrome. Am J Med. 1982;73:77-81.

3. Madhwal S, Goldberg J, Barcena J, Guha A, Gogate P, Cmolik B, et al. Unusual cause of acute mitral regurgitation: idiopathic hypereosinophilic syndrome. Ann Thorac Surg. 2012;93:974-7.

4. Mizota T, Miyawaki I, Enoki T, Nakao S. Massive prosthetic valve thrombus in a patient with idiopathic hypereosinophilic syndrome. J Cardiothorac Vasc Anesth. 2007;21:434-5.

5. Watanabe K, Tournilhac O, Camilleri LF. Recurrent thrombosis of prosthetic mitral valve in idiopathic hypereosinophilic syndrome. J Heart Valve Dis. 2002; 11:447-9.

\title{
Development of a life-threatening mediastinal hematoma from a ruptured bronchial artery aneurysm
}

\author{
Hee Jung Kim, MD, ${ }^{a}$ Ho Sung Son, MD, PhD, ${ }^{a}$ Sung Bum Cho, MD, PhD, ${ }^{b}$ and Kwang Taik Kim, MD, \\ $\mathrm{PhD},{ }^{\mathrm{a}}$ Seoul, South Korea
}

\footnotetext{
From the Department of Thoracic and Cardiovascular Surgery, ${ }^{\mathrm{a}}$ College of Medicine, Korea University, Seoul, South Korea; and the Department of Radiology, ${ }^{b}$ College of Medicine, Korea University, Seoul, South Korea.

Disclosures: Authors have nothing to disclose with regard to commercial support.

Received for publication June 4, 2013; revisions received July 1, 2013; accepted for publication July 12, 2013; available ahead of print Sept 11, 2013

Address for reprints: Ho Sung Son, MD, PhD, Department of Thoracic and Cardiovascular Surgery, College of Medicine, Korea University, 73 Inchon-ro, Sungbuk-gu, Seoul 136-705, South Korea (E-mail: hssonmd@korea.ac.kr).

J Thorac Cardiovasc Surg 2013;146:e47-9

$0022-5223 / \$ 36.00$

Copyright (C) 2013 by The American Association for Thoracic Surgery

http://dx.doi.org/10.1016/j.jtcvs.2013.07.034
}

Mediastinal bronchial artery aneurysm (BAA) is a rare condition. $^{1-4}$ It is detected in fewer than $1 \%$ of all patients who undergo selective bronchial arteriography. ${ }^{1}$ The clinical manifestation of BAA depends on size, location, and concomitant disease. ${ }^{4}$ Rupture can result in serious complications, such as life-threatening hemorrhage.

We report the case of a 79-year-old woman who was treated with transcatheter embolization and surgical removal of a mediastinal hematoma resulting from the life-threatening rupture of a BAA. 